\title{
Effect of Surfactants on Plasmid DNA Stability and Release from Poly (D,L-lactide-co-glycolide) Microspheres
}

\author{
Abd Almonem Doolaanea ${ }^{1,2 \star}$, Ahmad Fahmi Harun Ismail', Nur 'Izzati Mansor', \\ Nurul Hafizah Mohd Nor ${ }^{1}$ and Farahidah Mohamed ${ }^{1,2,3}$ \\ ${ }^{1}$ Department of Pharmaceutical Technology, ${ }^{2} I K O P$ Sdn. Bhd., Pilot Plant Pharmaceutical Manufacturing, Faculty of Pharmacy, \\ ${ }^{3}$ International Institute of Halal Research \& Training (INHART), Ground Floor, Block EO, Kulliyyah of Engineering, International \\ Islamic University Malaysia IIUM, PO Box 10, 50728, Kuala Lumpur, Malaysia
}

*For correspondence: Email: abdalmonemdoolaanea@yahoo.com; Tel: +60136238628; Fax: +609 5716775

Received: 9 May 2015

Revised accepted: 2 September 2015

\begin{abstract}
Purpose: To evaluate the effect of surfactants on plasmid DNA during preparation and release from polylactic glycolide (PLGA) microspheres.

Methods: Various surfactants, both ionic and non-ionic (Span, Tween, Triton X100, cetyltrimethylammonium bromide and sodium dodecyl sulphate), were added during the microsphere preparation and their effect was evaluated. Supercoil index (SCI) was introduced as a harmonised value derived from encapsulation efficiency and supercoil preservation efficiency in order to evaluate the impact of different surfactants on PDNA encapsulation.

Results: Polyvinyl alcohol and Span revealed low SCl whereas Tween increased the SCl in a fractiondependent manner. The Tween blend of hydrophilic-lipophilic balance (HLB) of 16 and Triton X-100 $(H L B=13.5)$ showed the highest SCI. Span revealed high burst release of pDNA whereas Triton X-100 exhibited low burst release. Following the burst release, diffusion mechanism was found to predominate in DNA release.

Conclusion: The microspheres were non-toxic to the neuro-2a cells which suggest they can be potentially used in the gene therapy of neuronal diseases.
\end{abstract}

Keywords: Surfactant, Gene therapy, Microspheres, Polylactic glycolide, Plasmid DNA, Supercoil index, Hydrophilic-lipophilic balance

Tropical Journal of Pharmaceutical Research is indexed by Science Citation Index (SciSearch), Scopus, International Pharmaceutical Abstract, Chemical Abstracts, Embase, Index Copernicus, EBSCO, African Index Medicus, JournalSeek, Journal Citation Reports/Science Edition, Directory of Open Access Journals (DOAJ), African Journal Online, Bioline International, Open-J-Gate and Pharmacy Abstracts

\section{INTRODUCTION}

Gene therapy remains attractive as a promising treatment approach for a wide range of diseases like cancer and allergies. A successful gene delivery system should be able to deliver nucleic acid efficiently to the target cell or tissue. Cell uptake of the gene delivery system and the release of the encapsulated nucleic acid are strongly related to the carrier physicochemical properties, which eventually will affect the transfection efficiency [1].
Solvent-evaporation method is a versatile technique to encapsulate macromolecules like DNA. The technique requires surfactant as the main component in the fabrication. A number of studies employing the technique have shown that surfactants had effects on the particle physical aging [2], internal structure [3] and morphological characteristics, [4].

However, to the best of our knowledge, there are no studies on the influence of surfactant on 
preservation of plasmid DNA ( $\mathrm{pDNA}$ ) integrity. It is a known fact that the super coil (SC) isoform of pDNA exerts the highest transfection efficiency [5]. High shear during microsphere fabrication by solvent-evaporation easily exposes pDNA to degradation detrimental to the efficacy of the gene delivery system. Reported attempts to preserve the pDNA integrity included freezing of the primary emulsion [6] and condensing the pDNA with cationic excipients $[7,8]$. Other excipients that have been also used to improve pDNA stability are lactose [6] and buffering agents such as $\mathrm{NaHCO}_{3}$ and $\mathrm{Na}_{2} \mathrm{HPO}_{4}$ [9].

In this study we encapsulated pDNA in Poly(D,Llactide-co-glycolide) (PLGA) microspheres with the focus on the role of surfactants in preserving the SC integrity of pDNA and attenuating the release properties.

\section{EXPERIMENTAL}

\section{Materials}

Plasmid DNA (pGL3-control vector) was purchased from Promega, Madison, WI. PLGA5004 (IV $0.4 \mathrm{dl} / \mathrm{g}$, ester-capped, of lactic to glycolic acid ratio of 50:50) was purchased from Purac, Gorinchem, Netherland. Polyvinyl alcohol (PVA) was purchased from BDH Chemicals, Poole, England. Cetyltrimethylammonium bromide (CTAB) and dichloromethane (DCM) were purchased from Sigma-Aldrich, Steinheim, Germany. Tween 20, Tween 80, Span 80, Span 85, Triton X100 (TX) and sodium dodecyl sulphate (SDS) were purchased from Merck, Hohen-brunn, Germany.

\section{Preparation and isoform analysis of pDNA}

Plasmid DNA was amplified in E. coli (Max Efficiency Dh5a, Invitrogen) and extracted using PureYield $^{\mathrm{TM}} \quad$ Plasmid Maxiprep System (Promega, Madison, WI) according to the manufacturer's protocol. The concentration and purity of pDNA were determined by measuring the absorbance at $260 \mathrm{~nm}\left(\mathrm{~A}_{260}\right)$ and the ratio $\mathrm{A}_{260} / \mathrm{A}_{280}$, respectively.

Following the extraction and purification steps, the pDNA was subjected to isoform analysis. Briefly, the purified pDNA together with $1 \mathrm{~kb}$ DNA ladder (GeneRuler ${ }^{\mathrm{TM}} 1 \mathrm{~kb}$ DNA Ladder, Fermentas) were loaded to $1 \% \mathrm{w} / \mathrm{v}$ agarose gel in 1X Tris-acetate-EDTA (TAE) buffer and run at $5 \mathrm{~V} / \mathrm{cm}$ for $90 \mathrm{~min}$. The agarose was then stained with $0.5 \mu \mathrm{g} / \mathrm{mL}$ ethidium bromide and the image was captured by Gel Documentation System
(Vilber Lourmat, France). The different isoforms of the plasmid migrated at different speeds in the gel resulting in separated bands after staining. The bands were further analysed using ImageJ software version $1.43 \mathrm{u}$ (US National Institute of Health) in order to integrate the optical density of each band. SC preservation of each lane is the optical density corresponding to the SC relative to the total optical density of all bands in the lane, expressed as percentage.

\section{Preparation of pseudo-primary emulsion}

Pseudo-primary emulsions were prepared to have similar ratio between components (water, DCM and surfactants) used in the microsphere fabrication. The word "pseudo" implied that PLGA and pDNA were not added to the oil and aqueous phases, respectively. $1 \% \mathrm{w} / \mathrm{v}$ (relative to the total volume of the emulsion) of the surfactants was added to a mixture of water:DCM $(1: 10)$ then the mixture was homogenised for $1 \mathrm{~min}$. Emulsion stability was evaluated by naked eyes wherein the time taken for the two phases to separate was recorded.

\section{Fabrication of pDNA-loaded PLGA microspheres}

Microparticles were prepared by a modified water-in-oil-in-water (W1/O/W2) double emulsion solvent-evaporation method as described before [10]. Briefly, pDNA in Tris-EDTA (TE) buffer (composing $\mathrm{W} 1$ phase) was added to the oil phase $(O)$ composed of PLGA 5004 in DCM then homogenised at 14,500 rpm for 1 min using IKA® T10 basic homogeniser (IKA Werke GmbH and Co., Staufen, KG, Germany). The resultant primary emulsion (W1/O) was immediately injected into $1 \%$ w/v PVA (W2) and homogenised for $3 \mathrm{~min}$ at the same speed to produce the secondary emulsion (W1/O/W2). The latter emulsion was immediately injected into a continuously stirred hardening tank constituted of $1 \% \mathrm{w} / \mathrm{v}$ PVA. Stirring was continued for $2 \mathrm{~h}$ to allow complete evaporation of the DCM. The hardened microspheres were then collected by centrifugation, washed three times with deionised water then lyophilised. Surfactants were added as $1 \% \mathrm{w} / \mathrm{v}$ of the primary emulsion. Span and Tween surfactants were blended together to generate a scale of HLB from 4.0 to 16.0. TX was used either alone $(H L B=13.5)$ or mixed with Span to yield HLB 10.0. SDS (HLB $=40)$ and CTAB $(H L B=10)$ were individually used (Table 1). 
Table 1: Blends of surfactants used in emulsion stability and microsphere formulation

\begin{tabular}{lll}
\hline No. & Surfactant(s) & HLB \\
\hline F1 & PVA & - \\
F2 & 88\% Span 80, 12\% Span 85 & 4.0 \\
F3 & 75\% Span 80, 25\% Tween 80 & 7.0 \\
F4 & 46\% Span 80, 54\% Tween 80 & 10.0 \\
F5 & 14\% Span 80, 86\% Tween 80 & 13.5 \\
F6 & 41\% Tween 80, 59\% Tween 20 & 16.0 \\
F7 & TX & 13.5 \\
F8 & SDS & 40.0 \\
F9 & CTAB & 10.0 \\
F10 & 38\% Span 80, 62\% TX & 10.0 \\
\hline
\end{tabular}

\section{Characterisation of pDNA-loaded PLGA microspheres}

\section{Particle size}

Particle size was measured prior to lyophilisation by laser diffraction sizing analyser (BT-9300H Dandong Bettersize Instruments, Dandong, China) and expressed as volume median diameter (D 0.5).

\section{Encapsulation and supercoil preservation efficiency}

An amount of $5-10 \mathrm{mg}$ of lyophilised microspheres was solubilised in chloroform and pDNA was extracted into TE buffer as described by Mohamed and van der Walle [4]. The aqueous layer was subjected to UV absorbance at $260 \mathrm{~nm}$ to determine pDNA concentration and encapsulation efficiency, and agarose gel electrophoresis for post-fabrication isoform analysis of pDNA.

\section{Supercoil index (SCI)}

$\mathrm{SCl}$ is proposed as a primary indicator of the quality of the pDNA-loaded microspheres. SCl is harmonised value from encapsulation efficiency and supercoil preservation efficiency, calculated by the following equation:

$\mathrm{SCl}=\mathrm{ESp} / 100$

where $E$ and $S p$ are encapsulation efficiency and supercoil preservation efficiency, respectively

$\mathrm{SCl}$ value indicates the amount of SC pDNA being encapsulated in the microspheres, making it more suitable to compare different microsphere formulations. As $\mathrm{SCl}$ is merely a calculated value, it was used only to compare different microsphere formulations. However, the effect of different variables was discussed separately for each of encapsulation efficiency and supercoil preservation efficiency.

\section{Thermal analysis}

Differential scanning calorimetry was conducted by a Differential Scanning Calorimeter (DSC1, STARe System, Mettler Toledo) using 2-3 mg of each sample in a standard $40 \mu \mathrm{L}$ aluminium crucible with pin-hole [11]. Both sample and reference (empty crucible with pin-hole) were left to equilibrate at $0{ }^{\circ} \mathrm{C}$ for $5 \mathrm{~min}$ (isothermal segment), heated to $85{ }^{\circ} \mathrm{C}$ (first heating), quench-cooled to $-20^{\circ} \mathrm{C}$ then re-heated again to $85^{\circ} \mathrm{C}$ (second heating). The first heating was conducted to eliminate the thermal history of the sample.

\section{Surface morphology}

Microspheres were sprinkled onto aluminium stub, pre-pasted with carbon adhesive tape. Samples were sputter-coated with gold and then viewed using Carl Zeiss Evo® 50 scanning electron microscope (SEM) (Oberkochen, Germany).

\section{Fluorescence microscopy}

Ethidium bromide was added to the pDNA solution prior to microsphere fabrication. Ethidium bromide binds to the double-strand pDNA producing fluorescence that can be detected under the fluorescence microscope. After fabrication, microspheres were suspended in deionised water in a 24-well plate and viewed under a Nikon Eclipse $\mathrm{Ti}$ fluorescence microscope.

\section{In vitro release studies}

Selected formulations were subjected to in vitro release study over a period of 4 weeks. Ten milligram of each formulation was suspended in $800 \mu \mathrm{L}$ TE buffer containing $0.02 \% \mathrm{w} / \mathrm{v}$ sodium azide and left undisturbed in a $37^{\circ} \mathrm{C}$ incubator. At a predetermined time intervals $(90 \mathrm{~min}, 5 \mathrm{~h}, 1$ day, 3 days and $1,2,3$ and 4 weeks), the microspheres were centrifuged and $600 \mu \mathrm{L}$ of the supernatant was withdrawn and replenished with equal volume of fresh TE buffer.

Concentration of pDNA was estimated by measuring the absorbance at $260 \mathrm{~nm}$ using UV spectrophotometer (Hitachi U1900 spectrophotometer, Japan). To study the stability of pDNA during the release period, $10 \mathrm{mg}$ of each of microsphere formulations was suspended in $800 \mu \mathrm{L}$ TE buffer then analysed by agarose gel after 90 min, 1 day, 1 week and 4 weeks. 


\section{Determination of cytotoxicity of microspheres}

Neuro 2a (N2a) cells were seeded in 96-well flatbottom microplate at a density of $2.5 \times 10^{5}$ cells per well and allowed to resume the growth by incubating the plate for $48 \mathrm{~h}$ at $37{ }^{\circ} \mathrm{C}$ and $5 \%$ $\mathrm{CO}_{2}$. The cells were treated with different microsphere formulations at different concentrations $(1,10,50,100$ and $500 \mu \mathrm{g} / \mathrm{mL})$. After $48 \mathrm{~h}$, the media were discarded and substituted with $30 \mu \mathrm{L}$ of $[3-(4, \quad 5-$ dimethylthiazole-2-yl)-5-(3-

carboxymethoxyphenyl)-2-(4-sulphonul)-2H-

tetrazolium] (MTT) reagent. The plate was then incubated for $4 \mathrm{~h}$. Following the incubation, 120 $\mu \mathrm{L}$ of DMSO was added and the absorbance of each well was read at $570 \mathrm{~nm}$ using Tecan Infinite 200 micro plate reader (Tecan Austria $\mathrm{GmbH}$, Grödig, Austria). The results were expressed as percentage relative to the control (untreated cells) which was assumed as $100 \%$ viable.

\section{Statistical analysis}

All data were obtained in triplicate and reported as mean \pm standard deviation (SD). One-way ANOVA with $95 \%$ confidence interval $(p<0.05)$ followed by Tukey post-hoc test was applied using Minitab (ver. 16) software (Minitab, Philadelphia, PA).

\section{RESULTS}

\section{Pseudo-primary emulsion stability}

As shown in Fig 1, stability of the emulsion increased as a function of HLB value for all sorbitan-based surfactants. TX with similar HLB (13.5) to that of Span-Tween blend gave lower stability, but the stability was comparable to $1 \%$ $\mathrm{w} / \mathrm{v}$ PVA. The latter has been commonly used in microencapsulation. In contrast, the cationic surfactant $C T A B$ resulted in similar stability to that of sorbitan-based surfactant blend of similar HLB while SDS seems to be a poor stabiliser.

\section{Effect of surfactants on pDNA-loaded PLGA microspheres}

\section{Microsphere particle size}

Based on the results shown in Table 2, it appears that all Span-containing microspheres tend to be larger in size than microspheres prepared by other surfactants. The size of the microspheres increased when Span fraction increased. Furthermore, adding Tween to the Span produced larger or similar microspheres size (F3 - F5). However, when Tween was used alone it produced microspheres with significantly smaller size (F6).

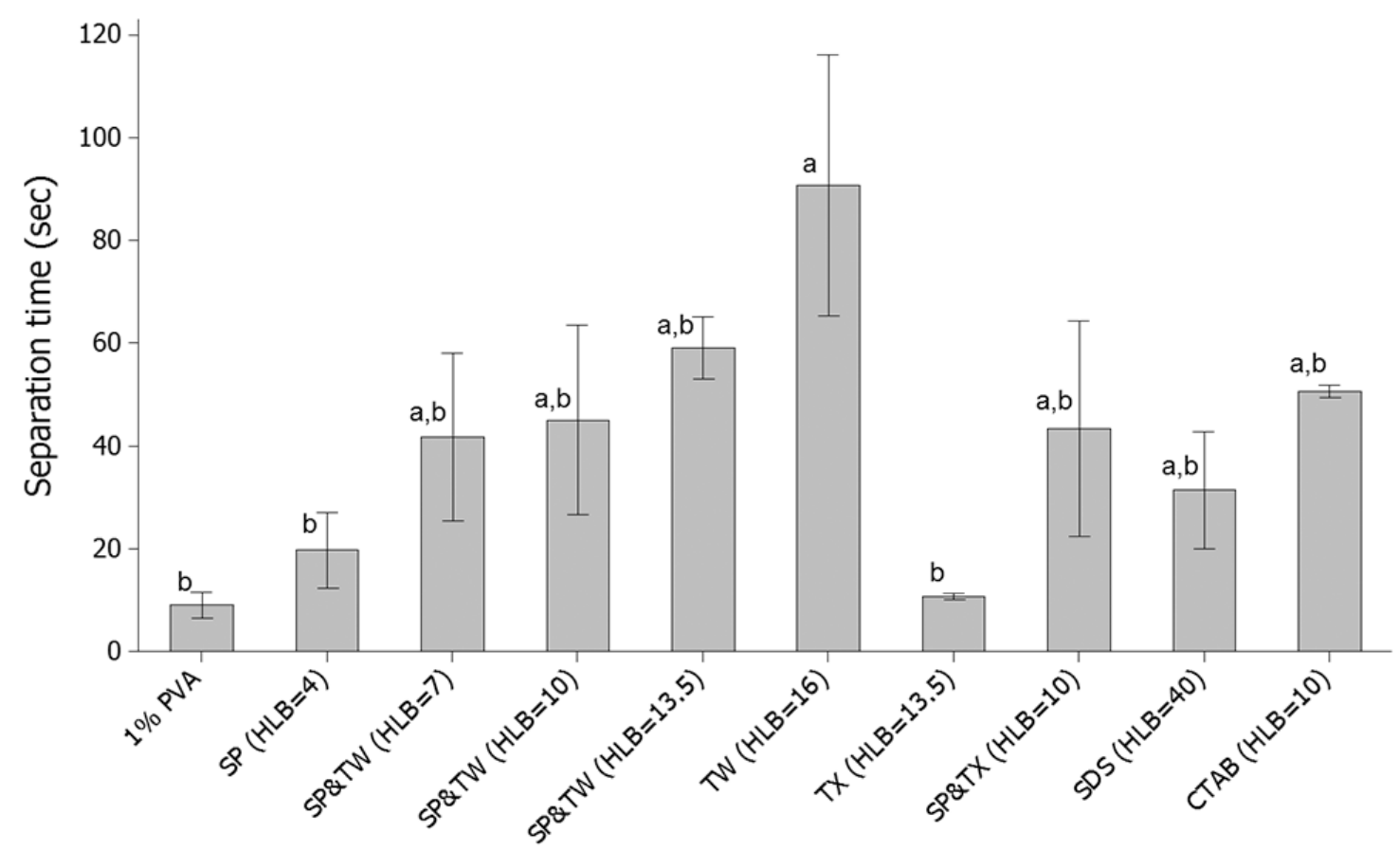

Surfactants

Figure 1: Screening of pseudo-primary emulsion stability 
Similarly, PVA which is a polymeric stabiliser produced smaller microspheres. Likewise, Triton $\mathrm{X}-100$ yielded comparable size to that of Tween and PVA despite its poor performance as stabiliser for the primary emulsion. CTAB (F9) yielded the smallest particle size while SDS produced significantly larger microspheres than CTAB.

\section{Microsphere encapsulation efficiency}

F6 formulation yielded the highest encapsulation efficiency and the most stable pseudo-primary emulsion (Table 2). The presence of hydrophobic Span surfactants in the formulation was deleterious to the encapsulation efficiency, as seen in F2-F5. This observation agrees with the results obtained by Mohamed and van der Walle [4] where it was shown that Tween 20 revealed higher pDNA encapsulation efficiency than Span 80. Although TX (HLB 13.5, F7) was a poor stabiliser of the pseudo-primary emulsion, it yielded similar encapsulation efficiency to that of Span-Tween microspheres (F3-F5). Similarly, when TX was blended with Span (F10), the encapsulation efficiency was compared to TX alone despite of the higher stability of the pseudo-primary emulsion. Interestingly, CTAB yielded similar encapsulation efficiency to that of Tweens (F6) although CTAB was poor stabiliser of pseudo-primary emulsion. In contrast, SDS was a poor stabiliser of the pseudo-primary emulsion and cannot complex the pDNA leading to low encapsulation efficiency.

\section{Plasmid stability (SC preservation) and glass transition $(\mathrm{Tg})$}

Degradation of pDNA from SC to other isoforms, mainly open circular (OC) occurred in all formulations with exception of F9 where SC preservation was not possible to be measured (Table 2; Fig 2). PVA seems to have a good stabilising effect on pDNA despite its low encapsulation efficiency. For F2-F6 which consisted of Span-Tween blends, it was found that the SC preservation was improved as HLB value increased i.e. as Tween fraction increased. Similar trend was also observed in the encapsulation efficiency. This could be attributed to the fact that SC isoform which has smaller size is more difficult to integrate with the polymer molecules. Consequently, SC pDNA can easily escape to the outer W2 phase during the fabrication process.

Table 2: Effect of different surfactants and surfactant blends on physical characteristics of pDNA-loaded PLGA microspheres. In each column, means that do not share a letter are significantly different $(p<0.05)$.

\begin{tabular}{lccccc}
\hline No. & $\begin{array}{c}\text { Particle size } \\
(\boldsymbol{\mu m})\end{array}$ & $\begin{array}{c}\text { Encapsulation } \\
\text { efficiency }(\%)\end{array}$ & $\begin{array}{c}\text { Supercoil } \\
\text { preservation }(\%)\end{array}$ & SCl & $\boldsymbol{T}_{\boldsymbol{g}}\left({ }^{\circ} \mathrm{C}\right)$ \\
\hline F1 & $10.4 \pm 0.02(\mathrm{~g})$ & $27.7 \pm 7.4(\mathrm{c}, \mathrm{d})$ & $71.7 \pm 10.1(\mathrm{a}, \mathrm{b})$ & $20.3 \pm 7.4(\mathrm{c}, \mathrm{d})$ & 42.4 \\
F2 & $18.3 \pm 0.07(\mathrm{c})$ & $13.9 \pm 5.3(\mathrm{~d})$ & $23.9 \pm 0.4(\mathrm{~d})$ & $3.3 \pm 1.2(\mathrm{e})$ & 40.5 \\
F3 & $27.8 \pm 0.08(\mathrm{~b})$ & $50.8 \pm 1.0(\mathrm{a}, \mathrm{b}, \mathrm{c})$ & $21.8 \pm 0.8(\mathrm{~d})$ & $11.1 \pm 0.5(\mathrm{~d}, \mathrm{e})$ & 40.6 \\
F4 & $17.7 \pm 0.24(\mathrm{~d})$ & $55.7 \pm 9.1(\mathrm{a}, \mathrm{b}, \mathrm{c})$ & $45.9 \pm 0.7(\mathrm{c})$ & $25.5 \pm 3.8(\mathrm{~b}, \mathrm{c}, \mathrm{d})$ & 41.1 \\
F5 & $17.0 \pm 0.10(\mathrm{e})$ & $68.6 \pm 7.9(\mathrm{a}, \mathrm{b})$ & $48.5 \pm 0.6(\mathrm{~b}, \mathrm{c})$ & $33.2 \pm 3.6(\mathrm{~b}, \mathrm{c})$ & 41.2 \\
F6 & $10.2 \pm 0.20(\mathrm{~g})$ & $75.9 \pm 5.3(\mathrm{a})$ & $67.5 \pm 7.9(\mathrm{a}, \mathrm{b})$ & $51.4 \pm 8.4(\mathrm{a})$ & 40.1 \\
F7 & $12.8 \pm 0.03(\mathrm{f})$ & $46.8 \pm 3.0(\mathrm{~b}, \mathrm{c})$ & $76.5 \pm 10.0(\mathrm{a})$ & $36.0 \pm 7.0(\mathrm{~b})$ & 40.9 \\
F8 & $9.2 \pm 0.02(\mathrm{~h})$ & $11.3 \pm 1.7(\mathrm{~d})$ & $31.8 \pm 10.8(\mathrm{c}, \mathrm{d})$ & $3.6 \pm 1.4(\mathrm{e})$ & 43.3 \\
F9 & $2.2 \pm 0.0(\mathrm{i})$ & $72.6 \pm 21.4(\mathrm{a}, \mathrm{b})$ & - & - & 42.4 \\
F10 & $29.9 \pm 0.49(\mathrm{a})$ & $47.4 \pm 14.7(\mathrm{~b}, \mathrm{c})$ & $82.7 \pm 9.4(\mathrm{a})$ & $38.3 \pm 7.0(\mathrm{a}, \mathrm{b})$ & 38.5 \\
\hline
\end{tabular}

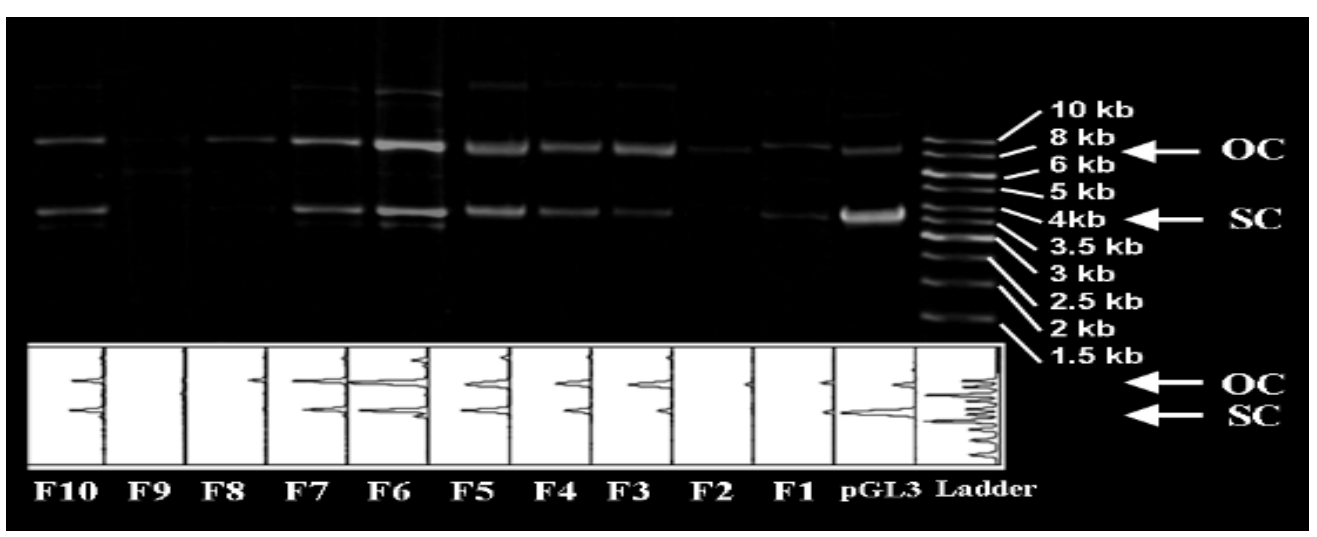

Figure 2: Agarose gel electrophoresis for the pDNA extracted from pDNA-loaded PLGA microspheres 
TX exhibited the highest preservation efficiency ( 77\%). Blending TX with Span significantly improved the preservation efficiency (F10 vs. F2). In other words, presence of Span in TX did not reduce the effect of TX on pDNA integrity unlike the combination between Span and Tween which reduced the preserving effect of the Tween. Additionally, Span-TX of HLB 10 (F10) revealed higher pDNA stability than Span-Tween of the same HLB value (F4). SDS appeared to confer some protective effect on pDNA but this was of little significant due to the low encapsulation efficiency. It was not possible to assess the preservation efficiency of CTAB (F9) because there was no corresponding band detected on the agarose gel.

\section{Supercoil index (SCl)}

Encapsulation efficiency represents the ability of a specific formulation to encapsulate pDNA regardless of its isoform whereas SC preservation efficiency represents the ability of the formulation to preserve the encapsulated plasmid in its SC isoform. While it is important to characterise the formulations for both encapsulation and SC preservation efficiency, individual value does not reflect the overall quality of specific formulation. Hence, to harmonise the two values, the so-called Supercoil Index (SCl) was introduced as a valuable initial indicator of a good gene delivery system. Based on $\mathrm{SCl}$, it is clearly seen that $\mathrm{F} 6$ has the highest $\mathrm{SCl}$, which indicates that Tween

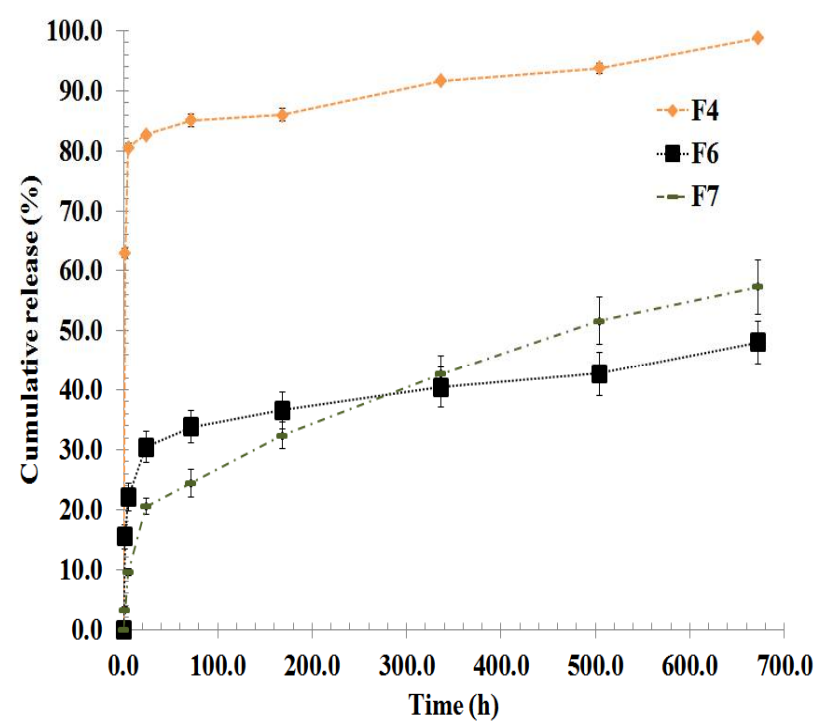

blend of HLB 16 could be used as a good gene delivery system.

\section{pDNA release profile}

Three formulations of different HLB values were selected for the release profile study. The low HLB (HLB 4 and HLB 7) formulations were not selected as they had low $\mathrm{SCl}$ and hence they are not good candidates as gene delivery carriers. For HLB 10 formulations (F4, F9 and F10) we selected F4 due its lower particle size. For HLB 13.5 ( $F 7$ and F10) we selected F7 due to its lower particle size and higher SC preservation. F6 was the only formulation of HLB 16 with good $\mathrm{SCl}$ and hence it was selected. SDS formulation (HLB 40, F8) was not selected because of the low SCl. The cumulative release at predetermined time from the three selected formulations is shown in Fig 3 while Fig. 4 shows the SEM images of the microspheres taken prior to incubation in dissolution media (day 0) and following $24 \mathrm{~h}$ and 28 days of incubation. The three formulations had smooth surface without visible pores as represented by Fig. 4 on Day 0. All microspheres exhibited varying amount of burst release in the first $5 \mathrm{~h}$, followed by sustained release phase over the 4 weeks. As repeatedly reported, the burst release was suggested to be due to the pDNA located near the surface [12]. Only F4 (Span-Tween blend of HLB 10) released more than $90 \%$ over the 4 weeks with more than $50 \%$ burst release in the first $5 \mathrm{~h}$.

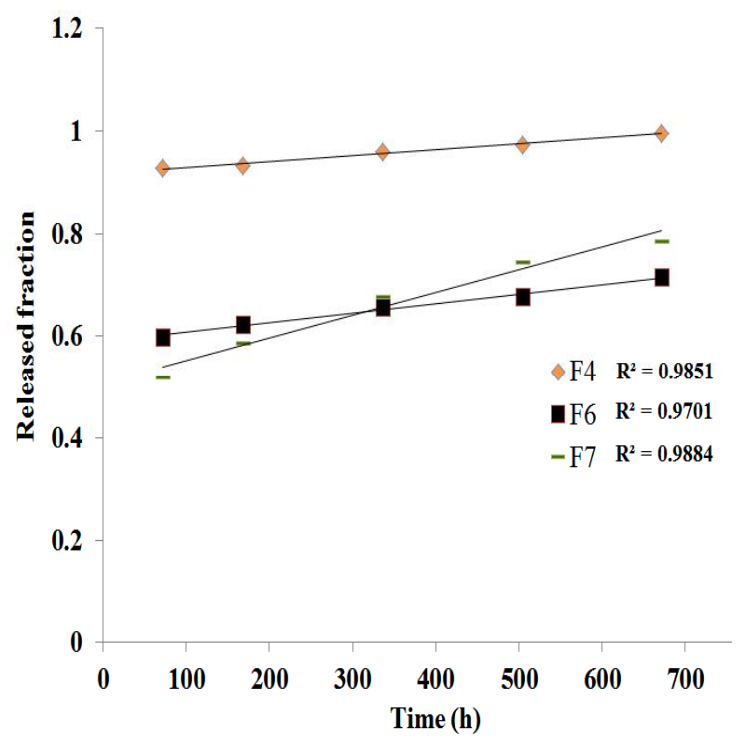

Figure 3: (A) Release profile of pDNA from pDNA-loaded PLGA microspheres. (B) Fitting the release profile kinetics on Higuchi model after excluding the burst release (24 h). 
Day (0)

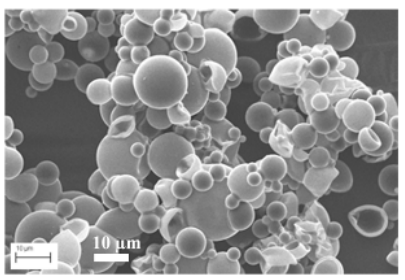

F4

(SP-TW, HLB 10)

F6

(TW, HLB 16)

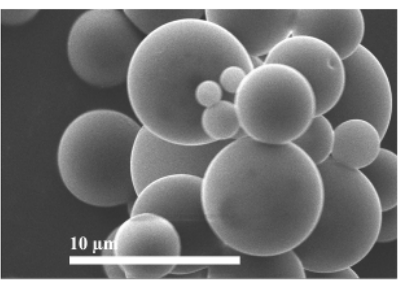

F7

(TX, HLB 13.5)
Day (1)
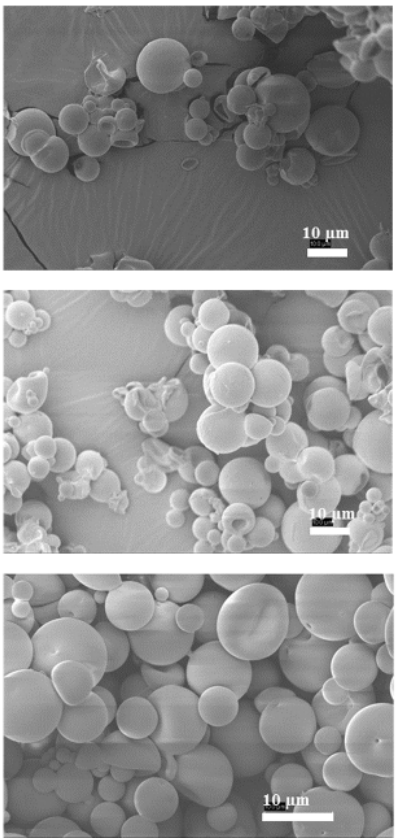

Day (28)
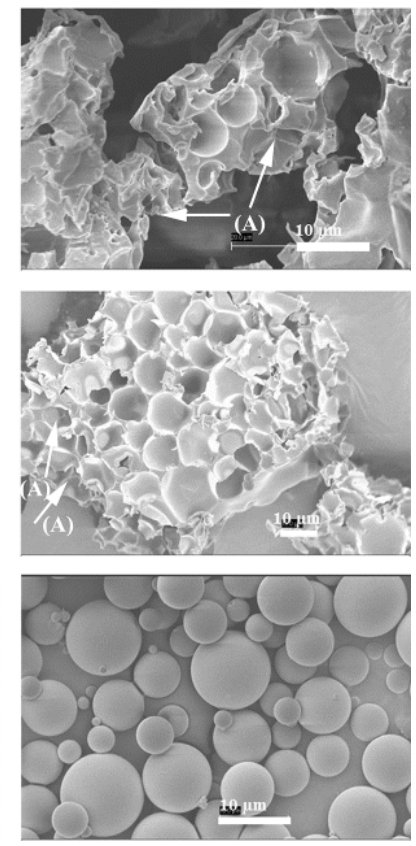

Figure 4: SEM images of pDNA-loaded PLGA microspheres before the release profile study (Day 0$)$ and following Day 1 and Day 28 of the release. Arrow $(A)$ denotes fractured microspheres

Fig 5 shows the stability of pDNA during the release study using agarose gel electrophoresis to detect the presence of pDNA and its conformation. Only the pDNA from F4 was detected on agarose gel in the first week (Fig. 6) while the released pDNA from F6 and F7 was not detected. However, after 4 weeks the pDNA was detected from F4 and F6 but not F7. The pDNA released after 4 weeks exhibited bands close to the position of the SC bands of the original pDNA. The bands after 4 weeks appeared broad, which can be related to the acidic environment and the high osmolarity of the release medium

due to PLGA degradation. The $\mathrm{pH}$ of the release medium was tested using Merck pH strips after 1 and 4 weeks and revealed that the media were acidic in the three formulations. After 1-week, media $\mathrm{pH}$ was 5-6 while after 4-weeks media $\mathrm{pH}$ was 4-5.

\section{Cytotoxicity of microspheres}

The microsphere formulations did not show significant cytotoxicity on N2a cells even at high concentration of $500 \mu \mathrm{g} / \mathrm{mL}$ (Fig 6).

1 week

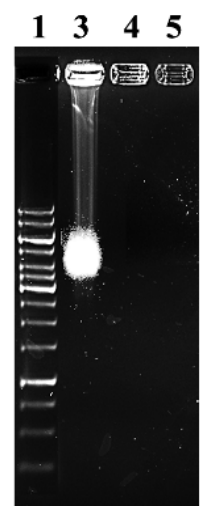

4 weeks

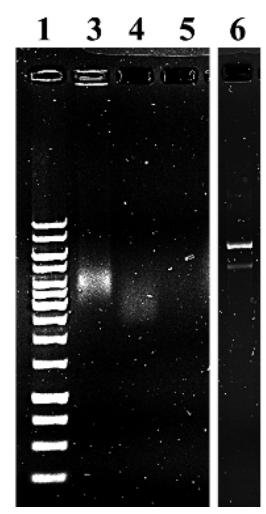

Figure 5: Stability of pDNA released from the microspheres as revealed by agarose gel electrophoresis. Lanes: (1) ladder, (2) pDNA used in the microsphere fabrication, (3) pDNA released from F4, (4) pDNA released from F6, (5) pDNA released from F7, (6) pDNA extracted from F7 after 4-week release 


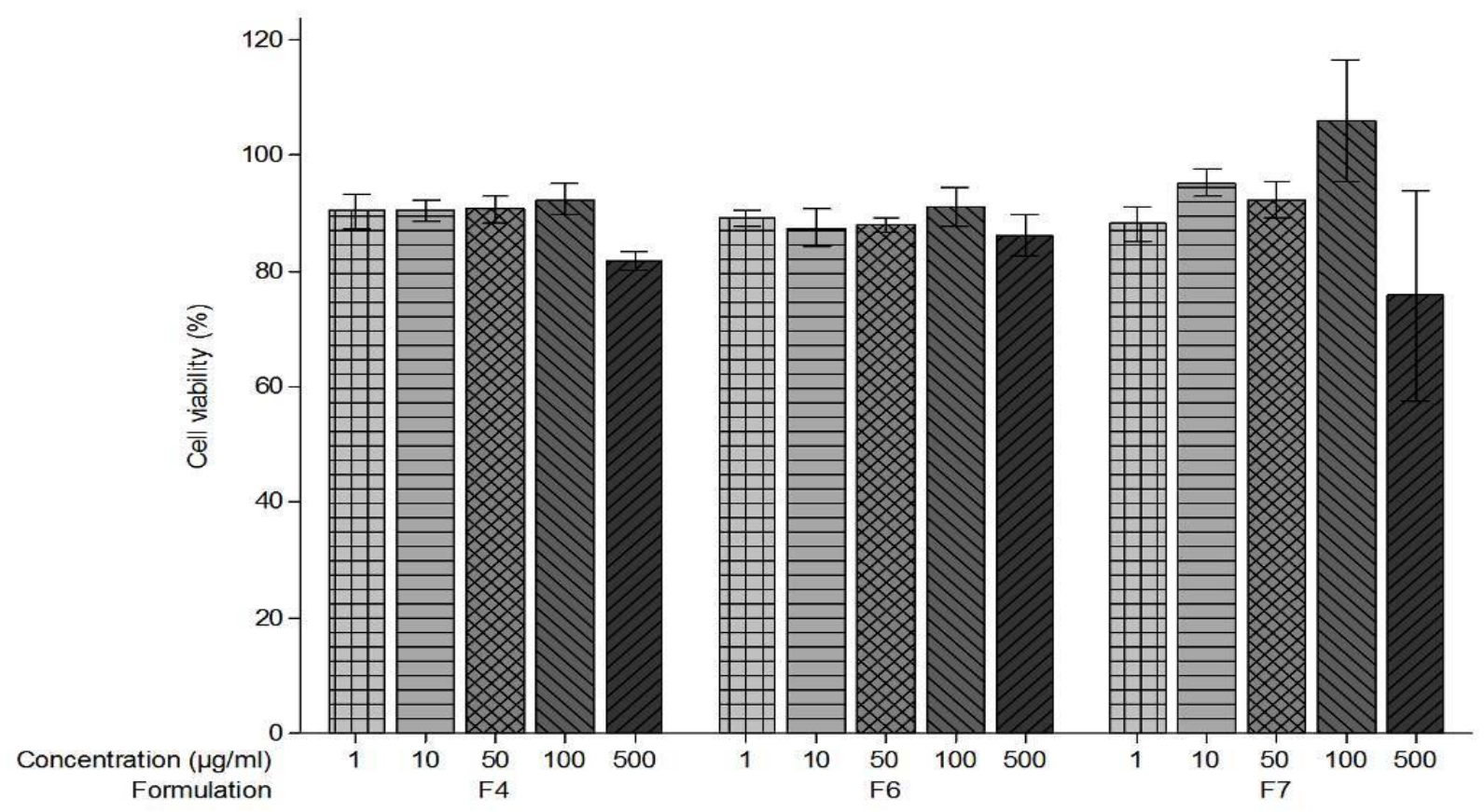

Figure 6: Cytotoxicity of the microspheres on N2a cells

\section{DISCUSSION}

Primary emulsion stability and polymer precipitation conditions play important roles in the encapsulation efficiency and the internal structure of the particles [13]. The notably small particle size of CTAB-PLGA microspheres may be attributed to the solubility of CTAB in both oil and aqueous phases as well as the electrostatic repulsion as observed in other studies $[11,14]$. In addition, the positively charged CTAB can interact with the negatively charged pDNA resulting in condensed structure that aids in producing smaller particles. The overall results suggest that the HLB and stability on the primary emulsion are not indicative of the microspheres size wherein surfactants of same HLB value resulted in microspheres with different size (F5 vs. F7, F4 vs. F9 \& F10). However, the stability of pseudo-primary emulsion was correlated to the encapsulation efficiency where F6 formulation yielded the highest encapsulation efficiency and the most stable pseudo-primary emulsion. The presence of hydrophobic Span surfactants in the formulation was deleterious to the encapsulation efficiency, as seen in F2-F5. This is because Span surfactants stabilise W/O emulsions and potentially induce the formation of reverse micelles. These reversed micelles transport the water and the water soluble pDNA from the internal $W 1$ to the external W2 phase through the thin layer of the oil phase [15].
It is believed that the SC isoform has the highest biological activity $[5,16,17]$; therefore, transformation of the SC to other isoforms like open circular (OC) or linear is considered as degradation. In this study, surfactants that function as emulsifiers have been evaluated for the potential use as pDNA protective agents. The mechanism of SC pDNA preservation by nonionic surfactants during microsphere fabrication has never been investigated before. Previous studies showed that the presence of the polymer (PLGA) in the oil phase was the main inducer for pDNA degradation upon the exposure to the shear force $[9,18]$. It was proposed that the hydrophobic vinyl acetate part of the partially hydrolysed PVA acted as anchors at the DCM interface. Once it is entangled with PLGA, it becomes very difficult to be removed from the surface $[19,20]$. These entanglements could trap the pDNA leading to conformational change in the pDNA due to the shear stress [9]. We postulate that the non-ionic surfactant added to the pDNA in W1 may have been adsorbed on the DNA strands by means of hydrogen bonding and other forces, hence making a protective 'envelope' around the pDNA. The envelope acts as a shield against the shear stress caused by PLGA and PVA molecules. This could be predicted especially for formulations containing Tween and TX whereby both surfactants have long hydrophilic polyoxyethylene side chains. This favourable interaction could be similar to the interaction between polyethylene glycol (PEG) 
and DNA, which results in precipitating of DNA molecules [21]. Microspheres prepared with Span alone (F2) did not show similar preservation efficiency. This could be due to the absence of a long hydrophilic chain to interact with the pDNA. Furthermore, Span seems to compromise the preservation efficiency of Tween but not TX. This could be explained by looking at the plasticising effect of these surfactants on PLGA, i.e. by comparing their $\mathrm{Tg}$. Lower $\mathrm{Tg}$ implies plasticising effect and indicates disruption of the polymer molecular arrangement. PVA was present in all formulations and accounted for the increased $\mathrm{Tg}$ of all microspheres ( $\mathrm{Tg}$ of 'raw' PLGA is $38.3^{\circ} \mathrm{C}$ ), indicating anti-plasticising effect. Consequently, when a surfactant results in $\mathrm{Tg}$ lower than that of PVA-containing microsphere (F1), this surfactant acts as stabiliser hence it compromises the interaction between PVA and PLGA. When Span and Tween were blended (F3-5, Table 1), the plasticising effect of the blend was lower than that when Span only (F2) or Tween only (F6) was used. On the other hand, blending Span with TX enhanced the plasticising effect of each of them (F10 compared to F7). This may be attributed to the tendency of Span and Tween molecules to enter in some kind of molecular association at the oil-water interface [22], hence reducing the amount of the surfactants in the PLGA matrix. It was reported that the emulsifier molecules cannot associate easily when the hydrocarbon chains contain less than 8 carbon atoms [22], the character that is not available in TX molecule (Fig. 1). Consequently, Span-TX blend exhibited better preservation efficiency than Span-Tween blends. By providing a protective envelope around DNA strands and compromising the PLGA and PVA molecular interactions, the non-ionic surfactants seems to act as lubricants that protect the labile pDNA from the shear force caused by PLGA and the entangled PVA molecules.

Even though the postulated mechanism seems to suggest that PVA is detrimental to the integrity of the pDNA, high SC preservation was observed in $\mathrm{F} 1$ when PVA was employed as a surfactant for W/O emulsion. It seems that PVA imparted its protective effect when it was added to the $\mathrm{W} 1$ phase in the primary emulsion.

The release of encapsulated substance from PLGA microspheres is governed by diffusion of the substance and degradation of the PLGA [23]. Different surfactants revealed apparently different release profiles. This can be partially attributed to the difference in hydrophobicity of the microspheres due to the different surfactant blends. The hydration of the particles was strongly influenced by the hydrophilicity of the surfactants, which enhances the degradation rate of the particles leading to faster release [2]. F4, which was relatively more hydrophobic than F6 and also had larger particle size, exhibited faster release with a very high burst release. SEM image of F4 displayed total collapse of the PLGA wall by 4th weeks of incubation. Additionally, the reverse micelle formation due to the presence of Span in F4 could attribute to the fast release of pDNA. The pDNA could be trapped in the reverse micelles, which become microchannels upon contact with water. This particular localisation together with the weak interaction between the hydrophilic pDNA and the hydrophobic PLGA resulted in the observed high burst release from F4. In contrast, F7 that contains TX seems to be very stable morphologically throughout the release period. The surfactants exhibited strong effect on the burst release (Fig. 3 A). However, after the burst release the three formulations exhibited release profile fitted properly on Higuchi model (Fig. 3 B) suggesting a diffusion controlled release mechanism in the sustained release phase [24].

Only the pDNA from F4 was detected on agarose gel in the first week. This can be attributed to the high burst release from F4. Since pDNA was successfully extracted from the remaining microspheres of F7 (lane 6 in Fig. 5), the absence of the released pDNA from $\mathrm{F} 7$ in agarose gel may be related to the slow release profile as shown previously in Fig. $3 \mathrm{~A}$. The pDNA seems to be stable after 4-weeks release period from the microspheres modified with different surfactants. In addition, the incorporated surfactants did not show significant cytotoxicity even though some of them such as TX is known to be highly toxic. In fact, TX is used in cell lysis buffers [25].

\section{CONCLUSION}

The findings of this study reveal the essential roles of surfactants not only as emulsifiers but also as protective agents to obtain sufficient supercoil pDNA from encapsulated PLGA microspheres. Supercoil index (SCl), as a harmonised value between encapsulation efficiency and supercoil preservation efficiency, is a useful indicator of the quality of microspheres. Among surfactants, the Tween blend of HLB 16 resulted in the highest SCI. The results also indicate that these surfactants can be used to protect pDNA and to modulate its 
release from PLGA microspheres without compromising the biocompatibility of the microspheres.

\section{ACKNOWLEDGEMENT}

This work was funded by Ministry of Science, Technology \& Innovation of Malaysia (MOSTI) (Grant ID: 02-01-08-SF0101).

\section{REFERENCES}

1. Luo D, Saltzman WM. Synthetic DNA delivery systems. Nat Biotechnol 2000; 18(1):33-37.

2. Bouissou C, Rouse JJ, Price R, van der Walle CF. The influence of surfactant on PLGA microsphere glass transition and water sorption: remodeling the surface morphology to attenuate the burst release. Pharm Res 2006; 23(6):1295-1305.

3. Dinarvand R, Moghadam SH, Sheikhi A, Atyabi F. Effect of surfactant HLB and different formulation variables on the properties of poly-D,L-lactide microspheres of naltrexone prepared by double emulsion technique. $J$ Microencapsul 2005; 22(2):139-151.

4. Mohamed F, van der Walle CF. PLGA microcapsules with novel dimpled surfaces for pulmonary delivery of DNA. Int J Pharm 2006; 311(1-2):97-107.

5. Li H, Bo H, Wang J, Shao H, Huang S. Separation of supercoiled from open circular forms of plasmid DNA, and biological activity detection. Cytotechnology 2011; 63(1):7-12.

6. Ando $S$, Putnam $D$, Pack DW, Langer R. PLGA microspheres containing plasmid DNA: Preservation of supercoiled DNA via cryopreparation and carbohydrate stabilization. J Pharm Sci 1999; 88(1):126-130.

7. Zhang $X Q$, Intra J, Salem AK. Comparative study of poly (lactic-co-glycolic acid)-poly ethyleneimine-plasmid DNA microparticles prepared using double emulsion methods. J Microencapsul 2008; 25(1):1-12.

8. Ravi Kumar M, Bakowsky U, Lehr C. Preparation and characterization of cationic PLGA nanospheres as DNA carriers. Biomaterials 2004; 25(10):1771-1777.

9. Tse MT, Blatchford C, Oya Alpar H. Evaluation of different buffers on plasmid DNA encapsulation into PLGA microparticles. Int J Pharm 2009; 370(1-2):33-40.

10. Doolaanea AA, Mansor NI, Mohd Nor NH, Mohamed F. Cellular uptake of Nigella sativa oil-PLGA microparticle by PC-12 cell line. J Microencapsul 2014; 31(6):600608.

11. Ismail H, Fahmi A, Doolaanea AM, Awang M, Mohamed $F$. High initial burst release of gentamicin formulated as PLGA microspheres implant for treating orthopaedic infection. International Journal of Pharmacy and Pharmaceutical Sciences 2012; 4(supp. 4):685-691.
12. Capan Y, Woo BH, Gebrekidan S, Ahmed S, DeLuca PP. Influence of formulation parameters on the characteristics of poly(d,l-lactide-co-glycolide) microspheres containing poly(l-lysine) complexed plasmid DNA. J Control Release 1999; 60(2-3):279286.

13. Nihant N, Schugens C, Grandfils C, Jerome R, Teyssie $P$. Polylactide microparticles prepared by double emulsion/evaporation technique. I. Effect of primary emulsion stability. Pharm Res 1994; 11(10):1479-1484.

14. Jeffery H, Davis SS, O'Hagan DT. The preparation and characterisation of poly(lactide-co-glycolide) microparticles. I: Oil-in-water emulsion solvent evaporation. Int J Pharm 1991; 77(2-3):169-175.

15. Nissim G. Double emulsions - scope, limitations and new achievements. Colloids Surf A Physicochem Eng Asp 1997; 123-124(0):233-246.

16. Sousa F, Prazeres DMF, Queiroz JA. Improvement of transfection efficiency by using supercoiled plasmid DNA purified with arginine affinity chromatography. J Gene Med 2009; 11(1):79-88.

17. Quaak SGL, den Berg JH, Oosterhuis K, Beijnen JH, Haanen JBAG, Nuijen B. DNA tattoo vaccination: effect on plasmid purity and transfection efficiency of different topoisoforms. J Control Release 2009; 139(2):153-159.

18. Walter E, Moelling K, Pavlovic J, Merkle HP. Microencapsulation of DNA using poly(dl-lactide-coglycolide): stability issues and release characteristics. $J$ Control Release 1999; 61(3):361-374.

19. Boury F, Ivanova T, Panaïotov I, Proust JE, Bois A, Richou J. Dynamic Properties of Poly(DL-lactide) and Polyvinyl Alcohol Monolayers at the Air/Water and Dichloromethane/Water Interfaces. J Colloid Interface Sci 1995; 169(2):380-392.

20. Lee SC, Oh JT, Jang MH, Chung SI. Quantitative analysis of polyvinyl alcohol on the surface of poly $(d, I-$ lactide-co-glycolide) microparticles prepared by solvent evaporation method: effect of particle size and PVA concentration. J Control Release 1999; 59(2):123-132.

21. Lis JT, Schleif $R$. Size fractionation of double-stranded DNA by precipitation with polyethylene glycol. Nucleic Acids Res 1975; 2(3):383-390.

22. Boyd J, Parkinson $C$, Sherman P. Factors affecting emulsion stability, and the HLB concept. J Colloid Interface Sci 1972; 41(2):359-370.

23. O'Donnell PB, McGinity JW. Preparation of microspheres by the solvent evaporation technique. Adv Drug Deliv Rev 1997; 28(1):25-42.

24. Costa P, Sousa Lobo JM. Modeling and comparison of dissolution profiles. Eur J Pharm Sci 2001; 13(2):123133.

25. Tinnikov AA, Samuels HH. A novel cell lysis approach reveals that caspase-2 rapidly translocates from the nucleus to the cytoplasm in response to apoptotic stimuli. PLoS One 2013; 8(4):e61085. 